\title{
Bitcoin: Bubble that bursts or Gold that glitters?
}

\begin{abstract}
This paper aims to shed light on the 2017 Bitcoin bubble. Firstly, by applying the dynamic time warping algorithm, we identify among several financial instruments a subsample of five assets with similar characteristics to the cryptocurrency bubble. Interestingly, among the fluctuations characterizing these assets, the algorithm shows a close affinity between the Bitcoin bubble and the 2000 NASDAQ Dotcom one. Once the subsample is identified, we study the (de)synchronization among these assets via the wavelet coherence approach. Although Bitcoin is poorly correlated with the other indices, given its scarce connection with the real economy, we observe switching phenomena among these instruments. A more careful study on these portfolio reallocations, conducted via an event study analysis, reveals that traders seem to redirect capital from stock markets and gold to Bitcoin in case of positive events of the cryptocurrency.
\end{abstract}

JEL codes: C58; G15

Keywords: Cryptocurrencies; bubbles; assets synchronization; hedging;

\section{Introduction}

It is now well established that cryptocurrencies, originally created to be free from the interference of governments or banks, are highly manipulable and volatile (Dodd, 2018). This appears especially true for Bitcoin, which proved to be particularly volatile in the face of external shocks driving traders to buy or sell large amounts of the cryptocurrency in short time periods. The economic literature showed how this cryptocurrency moved due to the effect of herding phenomena normally trigged by few traders. For instance, Gandal et al. (2018) demonstrated that, during the Bitcoin bubble of 2013, the cryptocurrency was manipulated by two investors who, with their trading activity, caused a significant increase in the Bitcoin price.

Other studies have shown the existence of a co-movement between the cryptocurrency price trend and the information published on social media (Urquhart, 2018). In this regard, a recent study has shown how positive and negative news have a strong positive impact on currency returns (Vidal-Tomas and Ibañez, 2018). Therefore, all these studies have confirmed how the presence of animal spirits can generate strong fluctuations in this currency dynamics via waves of optimism and pessimism. More generally, this "massuniform" behavior has been proven to be the key ingredient in affecting the currency price oscillation, which seems completely separated from any economic fundamental values (Baek and Elbeck, 2015). Following this prosperous research line, this manuscript aims to enrich the debate on the origins of cryptocurrency fluctuations and, in particular, to analyze two issues: i) are there coordination phenomena and similarities between the bitcoin fluctuations and those of other securities? ii) do switching phenomena emerge able to redirect traders' investments among different securities in case of news on Bitcoin? 


\section{The Data}

The goal of this manuscript is i) to compare the 2017 bitcoin speculative bubble with those of different financial instruments and ii)understand the reasons for the onset of this abnormal price hike. In this regard, we use the daily closing values of nine currencies exchange rates against US Dollars (i.e. EUR/USD, AUD/USD, CAD/USD, CHF/USD, JPY/ USD, GBP/USD, NZD/USD, NOK/ USD and SEK/ USD) and seven indices (STOXX50E, DAX30 CAC40, NASDAQ, S\&P500, DJI and XAU) ${ }^{1}$. Specifically, with respect to the first analysis, data run from 1/1/1999 to 30/11/2019 (5254 observations), while with respect to the second one they range from $15 / 9 / 2014$ to $30 / 11 / 2019$ (1310 observations).

\subsection{Bubbles comparison}

Figure. 1: DTW normalized distance from 2017 bitcoin bubble. The red box individuates the 5 closest shocks (left side). Alignment cost between Bitcoin and NASDAQ bubble (right side).
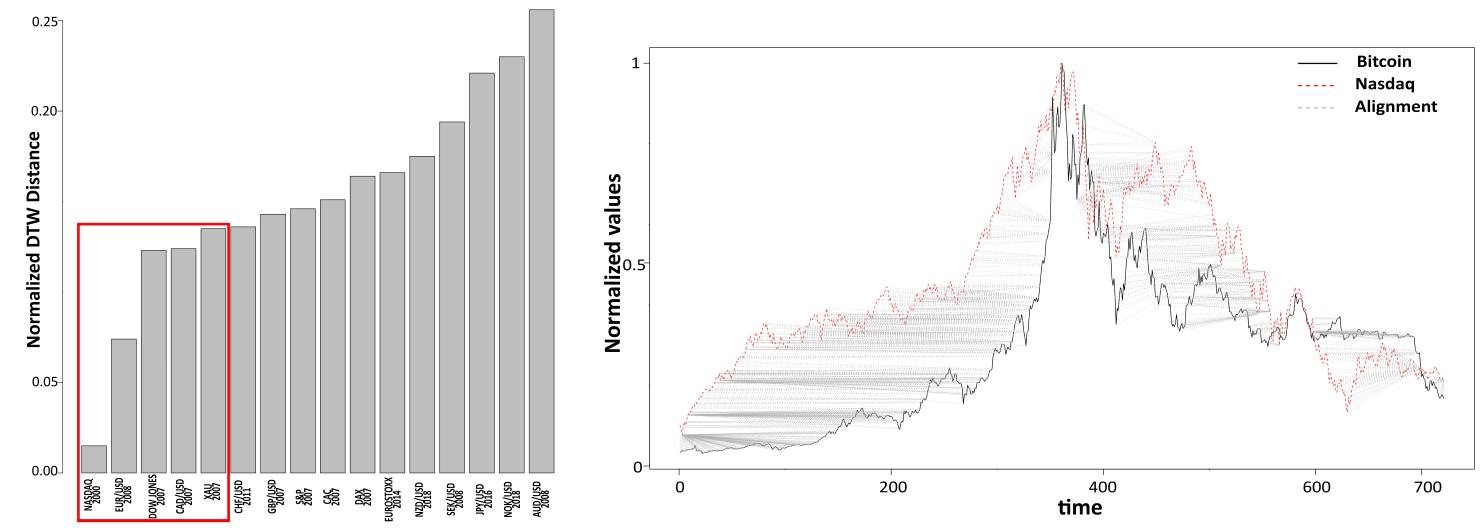

In order to compare different financial bubbles with the 2017 Bitcoin one, we apply the dynamic time warping algorithm (Bellman and Kalaba, 1959). This technique captures (dis)similarity between bitcoin and each one of the other investigated time series, by computing their optimal match at the minimal alignment cost, that is calculated as the sum of absolute differences between the values of each pair of points of the two indices. The higher the alignment cost, the higher the distance/dissimilarity between the two time series. The starting point of our analysis is the identification, in each of the considered time series, of those phenomena of market value rapid escalation to be compared with the 2017 bitcoin one. To this end, we select in each series the shock phases with the highest amplitude identified through the sADF test (Phillips, Wu, and $\mathrm{Yu}$ 2011). Around these turbulent periods, we select windows of 721 observations, that is 360 points before and after the peak. Once the windows are identified and normalized ${ }^{2}$, we apply the dynamic time warping algorithm (DTW). The left side of Fig. 1 shows the distances between the shocks hitting each financial instrument with respect to bitcoin one ${ }^{3}$. Interestingly, the closest affinity is with the 2000 NASDAQ Dotcom bubble. This is also evident from the low alignment cost between the two shocks displayed in the right side of Fig. 1. The five indices whose shocks are closest to the Bitcoin bubble are enclosed in the

\footnotetext{
${ }^{1}$ Data are downloadable from Yahoo Finance and Investing.com. In case of missing data, we control if values are missing completely at random (MCAR).

${ }^{2}$ During this phase, each time series observation $(\mathrm{x})$ has been normalized according to the following formula: $x_{\mathrm{norm}}=$ $x-\max (x) /(\max (x)-\min (x))$.

${ }^{3}$ The normalized DTW distance is the cumulative point alignment cost normalized by the time series lengths (see the R DTW package documentation, for details).
} 
red box in Fig. 1, left side (see supplementary materials: Tab. A for details ). In this list it is worthy of note how gold (XAU) and some currencies (CAD and EUR) shine. On the one hand, the affinity with the NASDAQ shock highlights that even the bitcoin bubble incorporates those animal spirits already present in the Dotcom bubble (Shiller, 2015 and Griffin et al. 2011). On the other hand, the incorporation of gold and currencies might suggest the traders propensity to diversify risk, since these financial instruments represent safe-haven solutions in case of extreme market shocks (Baur and McDermott, 2010; Bouri et al., 2017; Fatum and Yamamoto, 2016).

The robustness of our results on shocks similarities is verified i) by varying the window width (previously made up of 721 observations) ii) using returns time series, instead of prices. ${ }^{4}$ Results are available in the supplementary material.

\subsection{Behind the Bitcoin bubble: synchronization and switching phenomena}

After identifying a sample made up of 5 indices, we study the relationships between bitcoin and these financial instruments around the 2017 bubble. On the one hand, we analyze whether there exist synchronization among these asses, a phenomenon that would highlight a general exuberance in the market, often induced by coordination of expectations and herding, and able to create fluctuations in prices. On the other hand, we study the existence of switching phenomena among these assets that would identify the greater attractiveness of one of these instruments compared to the others. Switching, often caused by the arrival of news, signals the traders propensity to diversify risk and identify some financial instruments as alternative safe-haven solutions in case of extreme market shocks.

Firstly, to investigate the (de)synchronization between bitcoin and each one of the other five financial assets, we use the wavelet coherence approach (see Torrence and Compo (1998), for mathematical details). This procedure compares the fluctuations (wavelets) of two time series over time. At each time step, the approach decomposes the time series into wavelets with different frequencies, known as scales. Specifically, at small (large) scale values, high (low) frequency fluctuations correspond. Fig. 2 shows the synchronization/coherence between Bitcoin and the other series as a function of time and frequencies/scales. Synchronization increases (decreases) the more the colors tend to red (blue). Moreover, arrows indicate the correlation sign: arrows pointing to the right (left) indicate a positive (negative) correlation. At high frequency, results show a general lack of synchronization between these instruments and Bitcoin. This fact has a twofold explanation: 1) it reinforces the general idea of a poor connection of the cryptocurrency with the real economy (Caferra and Vidal-Tomas, 2021); 2) it indicates portfolio hedging and risk diversification (Bredin et al., 2015). At low frequency, the financial assets seem more synchronized. Specifically, we observe two interesting negative correlations: the first one between the Bitcoin and the euro exchange rate during the 2015-2017 weakening of the euro (EUR), and the second one in 2018 between the cryptocurrency and the Canadian dollar (CAD) in correspondence with the collapse of the former.

\footnotetext{
${ }^{4}$ Hereafter returns are calculated as $r_{t}=\left(P_{t}-P_{t-1}\right) / P_{t-1}$.
} 
Figure. 2: Wavelet coherence (synchronization) between bitcoin and Nasdaq, EUR/USD, DJI, CAD/USD and XAU, as a function of time and frequencies/scales. Synchronization increases (decreases) the more the colors tend to red (blue). Data run $15 / 9 / 2014$ to $30 / 11 / 2019$ (1310 daily observations).
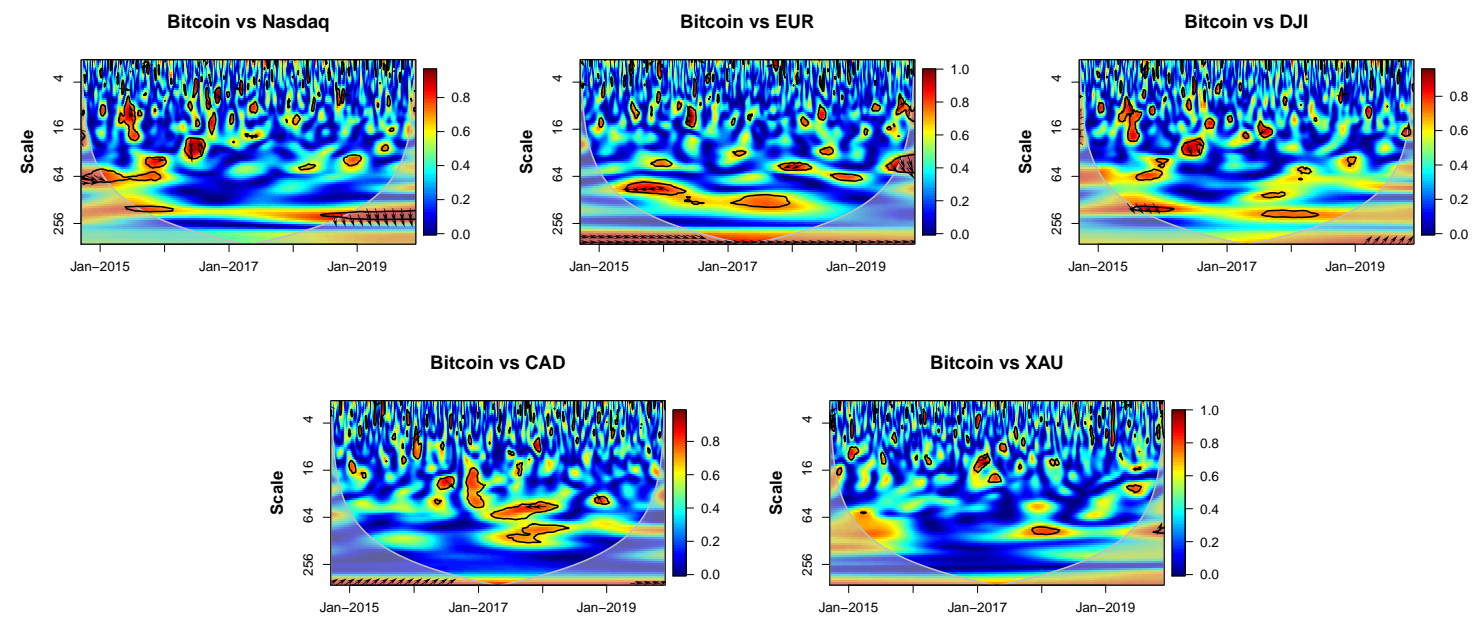

We now investigate if the arrival of good / bad news on the cryptocurrency generate switching phenomena among assets. We select a list of 36 cryptocurrency events and verify their impact on the series of risk-adjusted returns $^{5}$ using an ARFIMA (1,1,0)-fGARCH(1,1)-M model (Hentschel, L. 1995). The model specification is:

$$
\begin{gathered}
r_{t}=\mu+d+\alpha r_{t-1}+\eta \sqrt{h_{t}}+\beta_{2} p e_{t}+\beta_{3} n e_{t}+u_{t}, \\
u_{t} \mid \psi_{t-1} \sim i . i . d . N\left(0, h_{t}\right),
\end{gathered}
$$

where $r_{t}$ denotes the return of the stock index at day $\mathrm{t}, \mu$ and $d$ the simple average and the fractionally integrated moving average of returns, $\sqrt{h_{t}}$ the standard deviation of residuals, $p e_{t}$ and $n e_{t}$ dummy variables identifying positive and negative events and $u_{t}$ the error term. Moreover, $\psi_{t-1}$ is the past information conditioning the error term (Bera and Higgings 1993) and $h_{t}$ the conditional variance given by the ALLGARCH model. In order to grasp the anticipatory, instantaneous and future effect of news on markets returns, we estimate the model on the day before and after the announcement, that is the cumulative abnormal return (i.e $C A R(-1,1)$ ) where $p e_{t}$ and $n e_{t}$ are $\frac{1}{3}$ on days $t-1, t$, and $t+1$, and 0 otherwise. Results are shown in Tab. 1. Regarding the effect of news on cryptocurrency returns, we note that "the magnitude and influence of the negative events are evidently higher than the positive ones" (Feng et al., 2017). Furthermore, our results show the possible emergence of switching phenomena. Indeed, on the occasion of positive events regarding the cryptocurrency, we observe a decrease in the returns of the gold (XAU) and NASDAQ, which would suggest that investors move capital between the two markets. The same result also emerges in the case of negative shocks, although it is not statistically significant. Finally, the post-estimation test (P-E) on the time series returns indicates that all financial instruments are estimable with the chosen model.

\footnotetext{
${ }^{5}$ We employ the Fama-French (2015) five factors model
} 
Table 1: Regression results. Dependent Variable: Price returns. ${ }^{*}$ p-value $<0.1 ; * *$ p-value $<0.05 ; * * *$ p-value $<0.01$. Post estimation (P-E) heteroskedasticity and autocorrelation tests -ARCH and Ljung-Box $\mathrm{Q}^{2}$ tests.

\begin{tabular}{|c|c|c|c|c|c|c|}
\hline \multirow[b]{2}{*}{ Coefficients } & \multirow{2}{*}{$\begin{array}{c}\text { Crypotcurrency } \\
\text { BTC/USD }\end{array}$} & \multirow{2}{*}{$\begin{array}{l}\text { Gold } \\
\text { XAU }\end{array}$} & \multicolumn{2}{|c|}{ Stock } & \multicolumn{2}{|c|}{ Currency } \\
\hline & & & NASDAQ & DJI & CAD/USD & EUR/USD \\
\hline$\beta_{2}$ & $0.023(0.000)^{* * *}$ & $\overline{-0.018(0.008)^{* *}}$ & $-0.006(0.002)^{* *}$ & $-0.002(0.002)$ & $0.000(0.002)$ & 0.000 \\
\hline$\beta_{3}$ & $-0.029(0.012)^{* * *}$ & $0.006(0.006)$ & $0.000(0.001)$ & $-0.001(0.002)$ & $0.000(0.001)$ & $-0.001(0.002)$ \\
\hline \multicolumn{7}{|c|}{ P-E test (p-value) } \\
\hline $\operatorname{ARCH}(5)$ & 0.528 & 0.181 & 0.430 & 0.252 & 0.813 & 0.904 \\
\hline $\mathrm{Q}^{2}(\mathbf{9})$ & 0.671 & 0.565 & 0.714 & 0.337 & 0.291 & 0.757 \\
\hline
\end{tabular}

\section{Concluding remarks}

In this paper we have analyzed some possible reasons behind the emergence of abrupt fluctuations in the Bitcoin value. By applying the dynamic time warping algorithm, we have first identified a subset of financial instruments with characteristics similar to the 2017 Bitcoin bubble. This exercise has signaled a great affinity between the cryptocurrency dynamics in the 2017 and the 2000 NASDAQ Dotcom bubble.

Within this subset of assets and around the 2017 bubble, we have then studied the synchronization between these series and the emergence of switching phenomena. On the one hand, a wavelet coherence approach has shown a general lack of synchronization between these instruments and Bitcoin, therefore revealing portfolio hedging. On the other hand, the event study has indicated that traders move capital from the gold (XAU) and NASDAQ to Bitcoin, therefore suggesting switching phenomena. 


\section{References}

[1] Baek, C. and Elbeck, M. (2015). Bitcoins as an investment or speculative vehicle? a first look. Applied Economics Letters, 22(1):30-34.

[2] Baur,D. G., McDermott, T.K.,2010. "Is gold a safe haven? International evidence", Journal of Banking \& Finance, Volume 34, Issue 8;

[3] Bellman R. and Kalaba R., 1959. On adaptive control processes, Automatic Control, IRE Transactions;

[4] Bera, A. K., \& Higgins, M. L. (1993). ARCH models: properties, estimation and testing. Journal of economic surveys, 7(4), 305-366.

[5] Bouri, E., Molnar, P., Azzi, G., Roubaud, D., Hagfors, L.,I., 2017. On the hedge and safe haven properties of Bitcoin: Is it really more than a diversifier?, Finance Research Letters;

[6] Bredin, D., Conlon, T., \& Potì, V. (2015). Does gold glitter in the long-run? Gold as a hedge and safe haven across time and investment horizon. International Review of Financial Analysis, 41, 320-328.

[7] Caferra, R., Vidal-Tomas, D., 2021. Who raised from the abyss? A comparison between cryptocurrency and stock market dynamics during the COVID-19 pandemic. Finance Research Letters, 101954.

[8] Dodd, N. (2018). The social life of bitcoin. Theory, culture \& society, 35(3):35-56.

[9] Fama, E. F., \& French, K. R. (2015). A five-factor asset pricing model. Journal of financial economics, 116(1), 1-22.

[10] Fatum, R. and Yamamoto, Y., 2016. Intra-safe haven currency behavior during the global financial crisis, Journal of International Money and Finance;

[11] Feng, W., Wang, Y., Zhang, Z., 2017. Informed trading in the bitcoin market. Finance Research Letters;

[12] Griffin, J. M., Harris, J. H., Shu, T., \& Topaloglu, S. (2011). Who drove and burst the tech bubble?. The Journal of Finance, 66(4), 1251-1290.

[13] Gandal, N., Hamrick, J., Moore, T., and Oberman, T. (2018). Price manipulation in the bitcoin ecosystem. Journal of Monetary Economics, 95:86-96.

[14] Hentschel, L. 1995. All in the family nesting symmetric and asymmetric GARCH models. Journal of Financial Economics;

[15] Phillips, P. C., Wu, Y., \& Yu, J. (2011). Explosive behavior in the 1990s Nasdaq: When did exuberance escalate asset values?. International economic review, 52(1), 201-226.

[16] Shiller, R. J., 2015. Irrational exuberance: Revised and expanded third edition. Princeton university press.

[17] Torrence, C., \& Compo, G. P. (1998). A practical guide to wavelet analysis. Bulletin of the American Meteorological society, 79(1), 61-78

[18] Urquhart, A. (2018). What causes the attention of bitcoin? Economics Letters, 166:40-44.

[19] Vidal-Tomas, D. and Ibanez, A., 2018. Semi-strong efficiency of Bitcoin, Finance Research Letters; 